\title{
SYMMETRY AND RESONANCE \\ IN \\ HAMILTONIAN SYSTEMS
}

\author{
J. M. Tuwankotta* \\ F. Verhulst \\ Mathematisch Instituut \\ PO Box 80.010, 3508 TA Utrecht, \\ The Netherlands
}

November 23, 1999

\begin{abstract}
In this paper we study resonances in two degrees of freedom, autonomous, hamiltonian systems. Due to the presence of a symmetry condition on one of the degrees of freedom, we show that some of the resonances vanish as lower order resonances. After determining the size of the resonance domain, we investigate this order change of resonance in a rather general potential problem with discrete symmetry and consider as an example the Hénon-Heiles family of hamiltonians. We also study a classical example of a mechanical system with symmetry, the elastic pendulum, which leads to a natural hierarchy of resonances with the $4: 1$-resonance as the most prominent after the $2: 1$-resonance and which explains why the $3: 1$-resonance is neglected.
\end{abstract}

Keywords. Hamiltonian mechanics, higher-order resonance, normal forms, symmetry, elastic pendulum.

AMS clasification. 34E05, 70H33, 70K 30

\section{Introduction}

Symmetries play an essential part in studying the theory and applications of dynamical systems. For a general dynamical systems reference see [3], for symmetry in the context of hamiltonian systems see [4] and also [1], or [12]. In the older literature, attention was usually payed to the relation between symmetry and the existence of first integrals

*On leave from Jurusan Matematika, FMIPA,Institut Teknologi Bandung, Ganesha no. 10, Bandung, Indonesia 
but recently the relation between symmetry and resonance, in particular its influence on normal forms has been explored, see [13] for references.

In our analysis we shall be using a small parameter $\varepsilon$ which is introduced by re-scaling the variables. The implication is that, as $\varepsilon$ is small we analyze the dynamics of the hamiltonian flow in the neighborhood of equilibrium corresponding with the origin of phasespace. Note that $\varepsilon^{2}$ is a measure for the energy with respect to equilibrium. Putting $\varepsilon=0$, the equations of motion reduce to linear decoupled oscillators.

An important tool to analyze hamiltonian systems are normal forms. Based on the lowest degree of the expanded hamiltonian function where resonant terms are found, one can classify the resonances into three classes, i.e. first order, second order and higher order resonance. First order and second order resonances of two degrees of freedom have been considered intensively in the literature while higher order resonance has been considered in [6] and [7]. One can also classify resonance in the sense of energy interchange between the degrees of freedom. Terms like strong (or genuine) resonance and weak resonance are used to express the order of energy interchange on a certain time-scale which is characteristic for the dynamics of the system.

In this paper we are focusing on resonance in the presence of symmetry, see [13] for an introduction. In section 2 we will indicate how symmetry assumptions affect resonance and the normal forms. We use Birkhoff-Gustavson normalization which is equivalent with averaging techniques. In section 3 we give a sharp estimate of the size of the resonance domain at higher order resonance. Section 4 focuses on a special resonance, the $1: 2$-resonance for symmetric potential problems; we discuss an example from an important family of potential problems. Section 5 discusses one of the classical mechanical examples with symmetry, the elastic pendulum. In this problem, we show that the symmetry assumption produces a new hierarchy of resonances.

\section{Higher order resonance triggered by symmetry}

Consider the two degrees of freedom hamiltonian

$$
H\left(p_{1}, q_{1}, p_{2}, q_{2}\right)=\frac{1}{2} \omega_{1}\left(p_{1}^{2}+q_{1}^{2}\right)+\frac{1}{2} \omega_{2}\left(p_{2}^{2}+q_{2}^{2}\right)+H_{3}+H_{4}+\cdots .
$$

with $H_{k}, k \geq 3$ a homogeneous polynomial of degree $k$. Using action-angle transformation and Birkhoff-Gustavson normalization, we can transform the hamiltonian into normal form while preserving the hamiltonian structure. A hamiltonian $H$ is said to be in Birkhoff normal form of degree $2 k$ if it can be written as

$$
H=\omega_{1} \tau_{1}+\omega_{2} \tau_{2}+P_{2}\left(\tau_{1}, \tau_{2}\right)+P_{3}\left(\tau_{1}, \tau_{2}\right)+\cdots+P_{k}\left(\tau_{1}, \tau_{2}\right)
$$

where $P_{i}\left(\tau_{1}, \tau_{2}\right)$ is homogeneous polynomial of degree $i$ in $\tau_{j}=\frac{1}{2}\left(p_{j}{ }^{2}+q_{j}{ }^{2}\right), j=1,2$. The variables $\tau_{1}, \tau_{2}$ are called actions; note that if Birkhoff normalization is possible, the angles have been eliminated. If a hamiltonian can be transformed into Birkhoff normal form, the 
dynamics is fairly regular. The system is integrable with integral manifolds which are tori described by taking $\tau_{1}, \tau_{2}$ constant. The flow on the tori is quasi-periodic.

In normalizing, it is convenient if we transform to complex coordinates by

$$
\begin{aligned}
& x_{j}=p_{j}+i q_{j} \\
& y_{j}=p_{j}-i q_{j}, j=1,2,
\end{aligned}
$$

with corresponding hamiltonian $\tilde{H}=2 i H$.

The idea of Birkhoff-Gustavson normalization is to transform $H$ (we have dropped the tilde) so that the transformed hamiltonian becomes

$$
H\left(x_{1}, y_{1}, x_{2}, y_{2}\right)=\mathcal{B}_{k}\left(\tau_{1}, \tau_{2}\right)+R\left(x_{1}, y_{1}, x_{2}, y_{2}\right)
$$

where $\mathcal{B}_{k}$ is in Birkhoff normal form with $k$ as high as possible. $R$ is a polynomial which has degree of either $2 k$ or $2 k+1$ in $\left(x_{1}, y_{1}, x_{2}, y_{2}\right)$; it contains terms which can not be expressed in $\tau_{1}, \tau_{2}$ alone. The terms $R$ are also known as resonant interaction terms and $H$ in this form is called the Birkhoff-Gustavson normal form. In this paper we will refer to the terms in $R$ as resonant terms. For normalization one can use a generating function or suitable averaging techniques. See for example [1] appendix 8 or [12] chapter 11.

The presence of resonant terms of the lowest degree in the hamiltonian determines until what order the normalization should be carried out. For example, consider a general hamiltonian (1) and assume there is a pair of natural numbers $(m, n)$ such that $\frac{m}{n}=\frac{\omega_{1}}{\omega_{2}}$ where $m$ and $n$ are relatively prime. The resonant terms of the lowest degree are generally found in $H_{m+n} ; \omega_{1}: \omega_{2}$ is said to be a lower order resonance if the corresponding resonant terms of the lowest degree are found in $H_{k}$ with $k<5$.

It turns out that some of the lower order resonances are eliminated by symmetry in which case $m$ and $n$ need not be relative prime. We write the normal form (2) as,

$$
H\left(x_{1}, y_{1}, x_{2}, y_{2}\right)=\mathcal{B}_{k}\left(\tau_{1}, \tau_{2}\right)+2 i\left(D x_{1}{ }^{n} y_{2}{ }^{m}+\bar{D} y_{1}{ }^{n} x_{2}{ }^{m}\right)+\cdots .
$$

where $\tau_{j}=\frac{1}{2} x_{j} y_{j}, j=1,2$. In table 1 we present a list of lower order resonances and its corresponding resonant terms of the lowest degree. The second column shows resonant terms in a general hamiltonian system while the third column is for a hamiltonian system with symmetry in the second degree of freedom, i.e. $H\left(p_{1}, q_{1},-p_{2},-q_{2}\right)=H\left(p_{1}, q_{1}, p_{2}, q_{2}\right)$. Except for the $1: 1$ and $2: 1$-resonances, the other resonances are affected by the symmetry assumption. For example, the $1:$ 2-resonance in the general hamiltonian has resonant terms of the form $x_{1}^{2} y_{2}$ or $x_{2} y_{1}^{2}$. These terms vanish because of the symmetry assumption. Thus, instead of these terms which arise from $\mathrm{H}_{3}$, the resonant terms in the normal form derive from $H_{6}$ in the form of $x_{1}^{4} y_{2}^{2}$ or $x_{2}^{2} y_{1}^{4}$. We introduce the small parameter $\varepsilon$ by rescaling $x_{i}=\varepsilon \bar{x}_{i}, y_{i}=\varepsilon \bar{y}_{i}$ and divide the hamiltonian by $\varepsilon^{2}$. The normal form of the hamiltonian in the $1: 2$-resonance with discrete symmetry in the second degree of freedom is

$$
\begin{aligned}
H\left(x_{1}, y_{1}, x_{2}, y_{2}\right)= & i\left\{\left[\tau_{1}+2 \tau_{2}\right]+\varepsilon^{2}\left[A_{1} \tau_{1}^{2}+A_{2} \tau_{1} \tau_{2}+A_{3} \tau_{2}^{2}\right]+\right. \\
& \left.\varepsilon^{4}\left[B_{1} \tau_{1}^{3}+B_{2} \tau_{1}^{2} \tau_{2}+B_{3} \tau_{1} \tau_{2}^{2}+B_{4} \tau_{2}^{3}\right]+\varepsilon^{4}\left[D x_{1}^{4} y_{2}^{2}+\bar{D} y_{1}^{4} x_{2}^{2}\right]\right\}+\cdots .
\end{aligned}
$$




\begin{tabular}{|c|c|c|}
\hline \multirow{2}{*}{$\omega_{1}: \omega_{2}$} & \multicolumn{2}{|c|}{ Resonant term } \\
\cline { 2 - 3 } & General Hamiltonian & Symmetric in $x_{2}, y_{2}$ \\
\hline $1: 2$ & $x_{1}{ }^{2} y_{2}, x_{2} y_{1}{ }^{2}$ & $x_{1}{ }^{4} y_{2}{ }^{2}, x_{2}{ }^{2} y_{1}{ }^{4}$ \\
$2: 1$ & $x_{2}{ }^{2} y_{1}, x_{1} y_{2}{ }^{2}$ & $x_{2}{ }^{2} y_{1}, x_{1} y_{2}{ }^{2}$ \\
$1: 3$ & $x_{1}{ }^{3} y_{2}, x_{2} y_{1}{ }^{3}$ & $x_{1}{ }^{6} y_{2}{ }^{2}, x_{2}{ }^{2} y_{1}{ }^{6}$ \\
$3: 1$ & $x_{1} y_{2}{ }^{3}, x_{2}{ }^{3} y_{1}$ & $x_{1}{ }^{2} y_{2}{ }^{6}, x_{2}{ }^{6} y_{1}{ }^{2}$ \\
$1: 1$ & $x_{1}{ }^{2} y_{2}{ }^{2}, x_{2}{ }^{2} y_{1}{ }^{2}$ & $x_{1}{ }^{2} y_{2}{ }^{2}, x_{2}^{2} y_{1}{ }^{2}$ \\
& $x_{1}{ }^{2} y_{1} y_{2}, x_{1} x_{2} y_{2}{ }^{2}$ & \\
& $x_{1} y_{1}{ }^{2} x_{2}, y_{1} x_{2}{ }^{2} y_{2}$ & \\
\hline
\end{tabular}

Table 1: The table presents lower order resonant terms which cannot be removed by Birkhoff normalization. The second column shows resonant terms in the general case while in the third column we have added the symmetry condition $H\left(x_{1}, y_{1},-x_{2},-y_{2}\right)=$ $H\left(x_{1}, y_{1}, x_{2}, y_{2}\right)$.

The constants $D$ and $\bar{D}$ are complex conjugate.

It is also clear that symmetry in the second degree of freedom does not affect the $2: 1$ resonance. If we assume the symmetry is in the first degree of freedom, then this resonance will be affected while the $1: 2$-resonance will not. On the other hand, both the $3: 1$ - and $1: 3$-resonances are eliminated as a lower order resonance by the symmetry assumption, no matter on which degree of freedom the symmetric condition is assumed. As in mechanics one often has symmetries, this may also explain why these resonances received not much attention in the literature. This is demonstrated clearly for the elastic pendulum in section 5. For the 1 : 1-resonance, symmetry conditions on any degree of freedom (or even in both) do not push it into higher order resonance.

\section{The Resonance Domain}

Consider the normal form of a hamiltonian at higher order resonance

$$
H=\omega_{1} \tau_{1}+\omega_{2} \tau_{2}+\varepsilon^{2} P_{2}\left(\tau_{1}, \tau_{2}\right)+\cdots+\varepsilon^{m+n-2}\left(\tau_{1}^{n} \tau_{2}{ }^{m}\right)^{\frac{1}{2}} \cos (\chi),
$$

where $\chi=n \varphi_{1}-m \varphi_{2}+\alpha,\left(\tau_{1}, \varphi_{1}\right)$ and $\left(\tau_{2}, \varphi_{2}\right)$ are the action-angle variables of the first and the second degree of freedom, respectively, $m$ and $n$ are natural numbers satisfying $m+n \geq 5$ and $\frac{m}{n}=\frac{\omega_{1}}{\omega_{2}}$; and $\alpha \in[0,2 \pi)$. Note that $P_{k}$ is a homogeneous polynomial of degree $k$ and it corresponds to the $H_{2 k}$ term in the hamiltonian (1). Independent integrals of the system are $\mathcal{I}_{1}\left(\tau_{1}, \tau_{2}\right) \equiv \omega_{1} \tau_{1}+\omega_{2} \tau_{2}=E_{\circ}$, and $\mathcal{I}_{2}\left(\tau_{1}, \tau_{2}, \varphi_{1}, \varphi_{2}, \varepsilon\right) \equiv\left(H-\mathcal{I}_{1}\left(\tau_{1}, \tau_{2}\right)\right) / \varepsilon^{2}=C$.

In a seminal paper [6], Sanders described the flow on the energy manifold as follows. In the case of higher order resonance interesting dynamics takes place in the resonance domain which is imbedded in the energy manifold. The resonance domain contains a stable and an unstable periodic solution; the domain is foliated into tori on which the interaction 
between the two degrees of freedom takes place. In [6] the time-scale of the interaction is $\varepsilon^{-(m+n) / 2}$ and the size $d_{\varepsilon}$ of the resonance domain is estimated to be $O\left(\varepsilon^{(m+n-4) / 6}\right)$.

The estimate of $d_{\varepsilon}$ given in [6] is an upper limit, it depends on the approximation technique used there. Van den Broek [9](pp. 65-67) gave numerical evidence that the size of the resonance domain is actually smaller. In this section we shall present a sharp estimate of the size $d_{\varepsilon}$ which we derive from a Poincaré section of the flow.

The derivation runs as follows. First eliminate one of the actions, say $\tau_{1}$ using $\mathcal{I}_{1}$. Then we choose the section by setting $\varphi_{1}=0$. Thus we have a section in the second degree of freedom direction which is transversal to the flow of the system. For simplicity, we put $\alpha=0$ (otherwise we can rotate the coordinate with respect to the origin). The second integral $\mathcal{I}_{2}$ then becomes $\overline{\mathcal{I}_{2}}\left(\tau_{2}, \varphi_{2}, \varepsilon\right)=\mathcal{I}_{2}\left(\left(E_{\circ}-\omega_{2} \tau_{2}\right) / \omega_{1}, \tau_{2}, 0, \varphi_{2}, \varepsilon\right)$. Write

$$
\mathcal{P}(q, p, \varepsilon)=\overline{\mathcal{I}_{2}}\left(\frac{p^{2}+q^{2}}{2}, \arccos \left(\frac{q}{\sqrt{p^{2}+q^{2}}}\right), \varepsilon\right) .
$$

Fixing a value for $E_{\mathrm{o}}$, we draw the contour plot of (6) which gives us the Poincare map.

The contour plot of $\mathcal{P}$ in the $q-p$ plane (for a fixed $\varepsilon$ ) mainly consists of circles surrounding the origin. This is due to the fact that in the equations of motion, the equation for the actions are of order $\varepsilon^{m+n-2}$ and the equation for $\chi$ of order $\varepsilon^{2}$. This implies that for most of the initial conditions, the actions are constant up to order $\varepsilon^{m+n-2}$ and only the angles are varying. This condition fails to hold in a region where the righthand side of the equation for $\chi$ is zero or becomes small. Up to order $\varepsilon^{2}$, the location of this region can be found by solving

$$
n \frac{\partial P_{2}}{\partial \tau_{1}}-m \frac{\partial P_{2}}{\partial \tau_{2}}=0 .
$$

Note that in phase space, the equation above defines the so-called resonance manifold. On this resonance manifold there exist at least 2 short periodic solutions of the system (more if $m$ and $n$ are not relatively prime).

In the contour plot, these short periodic orbits appear as $2 m$ fixed points (excluding the origin) which are saddles and centers corresponding to the unstable and stable periodic orbit. Each two neighbouring saddles are connected by a heteroclinic cycle. Inside each domain bounded by these heteroclinic cycles, also known as the resonance domain, there is a center point. For example, see figure 3 in section 5 . We approximate the size of this domain by calculating the distance between the two intersection points of the heteroclinic cycle and a straight line $p=\lambda q$ for a $\lambda$ such that a center point is on the line.

Suppose we found one of the saddles $\left(q_{s}, p_{s}\right)$ and one of the centers $\left(q_{c}, p_{c}\right)$. We calculate $C_{\varepsilon}^{s}=\mathcal{P}\left(q_{s}, p_{s}, \varepsilon\right)$ and $C_{\varepsilon}^{c}=\mathcal{P}\left(q_{c}, p_{c}, \varepsilon\right)$. Note that since the integral $\mathcal{I}_{2}$ depends only on the actions up to order $\varepsilon^{m+n-4}$ we have $C_{\varepsilon}^{s}-C_{\varepsilon}^{c}=O\left(\varepsilon^{m+n-4}\right)$. The heteroclinic cycles are given by the equation $\mathcal{P}(q, p, \varepsilon)=C_{\varepsilon}^{s}$ and the intersection with the line $p=\lambda q$ is given by solving $\mathcal{P}(q, \lambda q, \varepsilon)=C_{\varepsilon}^{s}$. Write $q=q_{c}+\varepsilon^{\nu} \xi, \nu \in \mathbb{R}$. We want to determine $\nu$ which leads us to the size of the domain.

Note that $\mathcal{P}(q, \lambda q, \varepsilon)=\mathcal{P}_{4}(q, \lambda q)+\varepsilon^{2} \mathcal{P}_{6}(q, \lambda q)+\cdots+\varepsilon^{m+n-4} \mathcal{R}(q, \lambda q, \varepsilon)$, where $\mathcal{P}_{k}$ is a non-homogenous (in general) polynomial of degree $k$ and $\mathcal{R}$ is determined by the resonant 
term. Since

$$
\left.\left(\mathcal{P}^{\prime}\left(q_{c}, p_{c}, 0\right) \equiv\right) \frac{d}{d q} \mathcal{P}(q, \lambda q, \varepsilon)\right|_{\left(q_{c}, p_{c}, 0\right)}=0
$$

we expand $\mathcal{P}$ :

$$
\begin{aligned}
& \mathcal{P}_{4}\left(q_{c}, \lambda q_{c}\right)+\varepsilon^{2 \nu} \frac{1}{2} \mathcal{P}_{4}^{\prime \prime}\left(q_{c}, \lambda q_{c}\right) \xi^{2}+\cdots+ \\
& \quad \varepsilon^{2} \mathcal{P}_{6}\left(q_{c}, \lambda q_{c}\right)+\varepsilon^{2 \nu+2} \frac{1}{2} \mathcal{P}_{6}^{\prime \prime}\left(q_{c}, \lambda q_{c}\right) \xi^{2}+\cdots=C_{\varepsilon}^{c}+O\left(\varepsilon^{m+n-4}\right) .
\end{aligned}
$$

Since $\mathcal{P}_{4}\left(q_{c}, \lambda q_{c}\right)+\varepsilon^{2} \mathcal{P}_{6}\left(q_{c}, \lambda q_{c}\right)+\cdots+O\left(\varepsilon^{m+n-4}\right)=C_{\varepsilon}^{c}$, we have $\nu=(m+n-4) / 2$ and for the size of the resonance domain

$$
d_{\varepsilon}=O\left(\varepsilon^{\frac{m+n-4}{2}}\right)
$$

Of course degeneracies in the normal form may change this estimate. It is interesting to compare this with a formal method to derive the size of a resonance domain, described in [12], section 11.7. If we repeat the balancing method (method of significant degenerations) described there for our higher order resonance problem, we recover estimate (7).

\section{A potential problem with symmetry}

We will now study the $1: 2$ resonance in potential problems with a symmetry assumption. The normal form calculation is done using averaging techniques in a canonical way.

We rescale time to set one of the frequencies to be 1 ; we put $\omega_{1}=1$ and $\omega_{2}=\omega$. The hamiltonian with a potential, discrete symmetric in the second degree of freedom becomes

$$
\begin{aligned}
H= & \frac{1}{2}\left(\dot{q}_{1}^{2}+\dot{q}_{2}^{2}\right)+\frac{1}{2}\left(q_{1}^{2}+\omega^{2} q_{2}^{2}\right) \\
& -\varepsilon\left(\frac{1}{3} a_{1} q_{1}^{3}+a_{2} q_{1} q_{2}^{2}\right)-\varepsilon^{2}\left(\frac{1}{4} b_{1} q_{1}^{4}+\frac{1}{2} b_{2} q_{1}^{2} q_{2}^{2}+\frac{1}{4} b_{3} q_{2}^{4}\right) .
\end{aligned}
$$

Assume $\omega^{2}=4(1+\delta(\varepsilon))$. The reason for the assumption of the perturbation $\delta(\varepsilon)$ is that in applications we never encounter exact resonances; $\delta$ is an order function which is called the detuning to be specified later. In any case $\delta(\varepsilon)=o(1)$ as $\varepsilon \rightarrow 0$.

The induced equations of motion of hamiltonian (8) are

$$
\begin{array}{ll}
\ddot{q}_{1}+q_{1}=\varepsilon\left(a_{1} q_{1}{ }^{2}+a_{2} q_{2}{ }^{2}\right) & +\varepsilon^{2}\left(b_{1} q_{1}{ }^{3}+b_{2} q_{1} q_{2}{ }^{2}\right) \\
\ddot{q}_{2}+4 q_{2}=\varepsilon\left(2 a_{2} q_{1} q_{2}\right) & +\varepsilon^{2}\left(b_{2} q_{1}{ }^{2} q_{2}+b_{3} q_{2}{ }^{3}\right)-4 \delta(\varepsilon) q_{2} .
\end{array}
$$

The unperturbed form of system (9) (for $\varepsilon=0$ ) is linear and all solutions are periodic. The periodic solutions in one degree of freedom only, are called normal modes. The normal mode of the $p_{1}, q_{1}$ direction will be called the first normal mode and the other one will be called the second normal mode. Using averaging techniques, we will approximate other (short) periodic solutions up to order of $\varepsilon$ on some time-scale. Details of the averaging techniques and the asymptotic validity of the method can be found in [10] or [8]. 


\subsection{Lagrange standard form and averaging}

To apply the averaging techniques, we transform the equations of motion into amplitudephase form. Let

$$
\begin{array}{ll}
q_{1}=r_{1} \cos \left(t+\phi_{1}\right) & q_{2}=r_{2} \cos \left(2 t+\phi_{2}\right) \\
\dot{q}_{1}=-r_{1} \sin \left(t+\phi_{1}\right) & \dot{q}_{2}=-2 r_{2} \sin \left(2 t+\phi_{2}\right) .
\end{array}
$$

Using (10) we can transform (9) into the Lagrange standard form which has average zero to $O(\varepsilon)$. This means that on the time-scale $1 / \varepsilon$, both the amplitude and the phase are constant, up to order $\varepsilon$. If $\delta$ is of $O(\varepsilon)$ then there will be no fixed point in the averaged system and there is no interesting dynamics on this time-scale. Putting $\delta(\varepsilon)=\delta_{1} \varepsilon^{2}$, we perform second-order averaging which produces $O(\varepsilon)$ approximations on the time-scale $1 / \varepsilon^{2}$, see [8].

We find for the approximate amplitudes $\rho_{1}, \rho_{2}$ and phases $\varphi_{1}, \varphi_{2}$

$$
\begin{aligned}
& \dot{\rho}_{1}=0+O\left(\varepsilon^{3}\right) \\
& \dot{\varphi}_{1}=-\varepsilon^{2}\left(\left(\frac{5}{12} a_{1}^{2}+\frac{3}{8} b_{1}\right) \rho_{1}^{2}+\left(\frac{1}{2} a_{1} a_{2}+\frac{1}{15} a_{2}^{2}+\frac{1}{4} b_{2}\right) \rho_{2}^{2}\right)+O\left(\varepsilon^{3}\right) \\
& \dot{\rho}_{2}=0+O\left(\varepsilon^{3}\right) \\
& \dot{\varphi}_{2}=-\varepsilon^{2}\left(\left(\frac{1}{4} a_{1} a_{2}+\frac{1}{30} a_{2}^{2}+\frac{1}{8} b_{2}\right) \rho_{1}^{2}+\left(\frac{29}{120} a_{2}^{2}+\frac{3}{16} b_{3}\right) \rho_{2}^{2}-\delta_{1}\right)+O\left(\varepsilon^{3}\right) .
\end{aligned}
$$

From system (11), we conclude that, up to order $\varepsilon$ the amplitude of the periodic solution is constant. This result is consistent with the result in [11].

\subsection{The resonance manifold}

We shall define a combination angle $\chi$ which reduces the dimension of the averaged system by one. Moreover, a lemma by Verhulst [7] (stated there without proof), can simplify the equation for the combination angle. We present this theorem in a slightly different form:

Lemma 4.1 Consider the real Hamiltonian

$$
H=\frac{1}{2}\left(\dot{q}_{1}^{2}+\dot{q}_{2}^{2}\right)+V\left(q_{1}, q_{2}\right)
$$

where $V\left(q_{1}, q_{2}\right)$ is analytic near $(0,0)$ and has a Taylor-expansion which starts with $\frac{1}{2}\left(\omega_{1}^{2} q_{1}^{2}+\right.$ $\left.\omega_{2}^{2} q_{2}^{2}\right)$. Then the coefficient of the resonant term $D$ in the Birkhoff-Gustavson normal form (3) of the hamiltonian can be chosen as a real number.

\section{Proof:}

Assume $\frac{\omega_{1}}{\omega_{2}}=\frac{m}{n}$ where $m, n \in I$ and the hamiltonian in potential form as assumed in the lemma. Writing $\dot{q}_{j}=p_{j}$ the hamiltonian can be expressed as

$$
H=\frac{1}{2} m\left(p_{1}^{2}+q_{1}^{2}\right)+\frac{1}{2} n\left(p_{2}^{2}+q_{2}^{2}\right)+\sum_{k=3}^{\infty} \tilde{V}_{k}\left(q_{1}, q_{2}\right)
$$


where $\tilde{V}_{k}$ is the $k$-th term of the Taylor expansion of $V$. Define a transformation to complex coordinates by $x_{j}=p_{j}+i q_{j}$ and $y_{j}=\bar{x}_{j}$. In these variables the hamiltonian becomes

$$
\tilde{H}=2 i\left\{\frac{1}{2}\left(\omega_{1} x_{1} y_{1}+\omega_{2} x_{2} y_{2}\right)+\sum_{k=3}^{\infty} i^{k} \tilde{V}_{k}\left(\frac{x_{1}-y_{1}}{-2}, \frac{x_{2}-y_{2}}{-2}\right)\right\} .
$$

This means that the coefficient of each term in $\tilde{V}_{k}$ is either real or purely imaginary. The normalized hamiltonian is

$$
\tilde{H}=2 i\left\{P\left(\tau_{1}, \tau_{2}\right)+\left(D_{\circ} x_{1}{ }^{n} y_{2}{ }^{m}+\bar{D}_{\circ} y_{1}{ }^{n} x_{2}{ }^{m}\right)+\cdots\right\}
$$

where $\tau_{j}=\frac{1}{2} x_{j} y_{j}$ and $P$ is a real polynomial of even degree. In the case where $D_{\circ}$ is purely imaginary, write $D_{\circ}=D i$ and write the normal form (12) as

$$
\tilde{H}=2 i\left\{P\left(\tau_{1}, \tau_{2}\right)+D\left(x_{1}^{n} y_{2}^{m}-y_{1}^{n} x_{2}^{m}\right)+\cdots\right\} \text {. }
$$

If $D_{\circ} \in \mathbb{R}$ the proof is complete by setting $D=D_{\circ}$.

- Generalization of this lemma is possible by considering a wider class of hamiltonians by allowing terms like $p_{2}^{s} q_{2}{ }^{k} q_{1}{ }^{l}$ ( $s$ a fixed natural number, $k$ and $l$ are natural numbers) to exist in the hamiltonian.

A consequence of lemma 4.1 is, that in the equations of motion derived from the normal form of the hamiltonian we have the combination angle $\chi=n \varphi_{1}-m \varphi_{2}+\alpha$ with $\alpha=0$. The phase-shift $\alpha$ will not affect the location of the resonance manifold, it will only rotate it with respect to the origin but it will affect the location of the periodic solutions in the resonance manifold.

Because of this lemma, define $\chi=4 \varphi_{1}-2 \varphi_{2}$. Then, the averaged equations become

$$
\begin{aligned}
& \dot{\rho}_{1}=0 \\
& \dot{\rho}_{2}=0 \\
& \dot{\chi}=\varepsilon^{2}\left(\gamma_{1} \rho_{1}{ }^{2}+\gamma_{2} \rho_{2}{ }^{2}-2 \delta_{1}\right)
\end{aligned}
$$

where $\gamma_{1}=-\frac{5}{3} a_{1}^{2}+\frac{1}{2} a_{1} a_{2}+\frac{1}{15} a_{2}^{2}-\frac{3}{2} b_{1}+\frac{1}{4} b_{2}$ and $\gamma_{2}=-2 a_{1} a_{2}+\frac{13}{60} a_{2}^{2}-b_{2}+\frac{3}{8} b_{3}$. By putting the right hand side of the last equation zero, the resonance manifold is given by

$$
\gamma_{1} \rho_{1}^{2}+\gamma_{2} \rho_{2}^{2}=2 \delta_{1}
$$

The resonance manifold is imbedded in the energy manifold and contains periodic solutions; because of lemma 3.1 we know the location, one is stable and one is unstable. By fixing the energy, we fix also $\rho_{1}$ and $\rho_{2}$ and approximate them with their initial value while $\chi\left(\varphi_{1}, \varphi_{2}\right)$ varies (except on the resonance manifold).

Using the approximate energy integral, i.e. $E_{0}=\frac{1}{2} \rho_{1}{ }^{2}+2 \rho_{2}{ }^{2}$, assuming $\gamma_{2} \neq 4 \gamma_{1}$ we can solve (14) for $\rho_{1}{ }^{2}$ and $\rho_{2}{ }^{2}$, i.e.:

$$
\rho_{1}^{2}=\frac{2 \gamma_{2} E_{0}-8 \delta_{1}}{\gamma_{2}-4 \gamma_{1}} \text { and } \rho_{2}^{2}=\frac{2 \delta_{1}-2 \gamma_{1} E_{0}}{\gamma_{2}-4 \gamma_{1}}
$$


We shall now discuss what happens at exact resonance $\left(\delta_{1}=0\right)$. It is clear that $0 \leq \rho_{1}^{2} \leq 2 E_{0}$, so that we have, $0 \leq \frac{2 \gamma_{2} E_{0}}{\gamma_{2}-4 \gamma_{1}} \leq 2 E_{0}$. The last inequality is equivalent with $\gamma_{1} \gamma_{2} \leq 0$. If $\gamma_{1}$ tends to zero, then the resonance manifold will be approaching the first normal mode. For $\gamma_{2}$ tending to zero, the resonance manifold approaches the second normal mode. We exclude now the equality and will consider only the resonance manifold in general position.

We summarize in a lemma:

Lemma 4.2 Existence of the resonance manifold in general position for exact resonance

Consider hamiltonian (8) with $\delta(\varepsilon)=0$. A resonance manifold containing periodic solutions of the equations of motion induced by this Hamiltonian exists if and only if $\gamma_{1} \gamma_{2}<0$. Those periodic solution are approximated by $x=\rho_{1}(0) \cos \left(t+\varphi_{1}(t)\right)$ and $y=\rho_{2}(0) \cos (2 t+$ $\left.\varphi_{2}(t)\right)$ where $\rho_{1}(0)$ and $\rho_{2}(0)$ satisfy $(15), \varphi_{1}$ and $\varphi_{2}$ are calculated by direct integration of the second and the fourth equation of (11).

- Using a specific transformation, we can derive the mathematical pendulum equation $\ddot{\chi}+\Omega \chi=0$ related to the system (11), see [7]. The fixed points $\dot{\chi}=0, \pi, \dot{\chi}=0$ of the mathematical pendulum equation determine the locked-in phases of the periodic solutions by setting $4 \varphi_{1}-2 \varphi_{2}=0$ or $4 \varphi_{1}-2 \varphi_{2}=\pi$. The first one corresponds with the stable periodic solutions and the second one with the unstable periodic solutions.

- From section 3 we know that the size of the resonance domain is $d_{\varepsilon}=O(\varepsilon)$, the time-scale of interaction is $O\left(\varepsilon^{-3}\right)$. Note that the size $d_{\varepsilon}$ is in agreement with the work of van den Broek in [9].

\subsection{Examples from the Hénon-Heiles family of hamiltonians}

An important example of hamiltonian (8), with $b_{1}=b_{2}=b_{3}=0$, is known as the HénonHeiles family of Hamiltonians, see [11]. The condition for existence of the resonance manifold in exact resonance in lemma 4.2 reduces to

$$
\left(-\frac{5}{3} a_{1}^{2}+\frac{1}{2} a_{1} a_{2}+\frac{1}{15} a_{2}^{2}\right)\left(-2 a_{1} a_{2}+\frac{13}{60} a_{2}^{2}\right)<0 .
$$

Assuming $a_{2} \neq 0$ to avoid decoupling, we introduce the parameter $\lambda=a_{1} / 3 a_{2}$. Using this parameter, the existence condition can be written as $\left(450 \lambda^{2}-45 \lambda-2\right)(360 \lambda-13) \leq 0$. Thus, the resonance manifold for the Hénon-Heiles family exists for $\lambda<-\frac{1}{30}$ or $\frac{13}{360}<\lambda<$ $\frac{2}{15}$. Note that for the Contopoulos problem $\left(a_{1}=0\right)$ the resonance manifold does not exist at exact resonance while in the original Hénon-Heiles problem $\left(a_{1}=1\right.$ and $\left.a_{2}=-1\right)$ the resonance manifold exists.

From this analysis, we know that for $\lambda=\frac{2}{15}$ the resonance manifold will coincide with the first normal mode. Since for $\lambda>\frac{2}{15}$ the resonance manifold does not exist, let $\lambda$ decrease on the interval $\left(-\infty, \frac{2}{15}\right]$. The resonance manifold moves to the second normal 
mode which it reaches at $\lambda=\frac{13}{360}$. After that the resonance manifold vanishes and then emerges again from the first normal mode when $\lambda=-\frac{1}{30}$. The resonance manifold then always exist and tends to the second normal mode as $\lambda$ decreases.

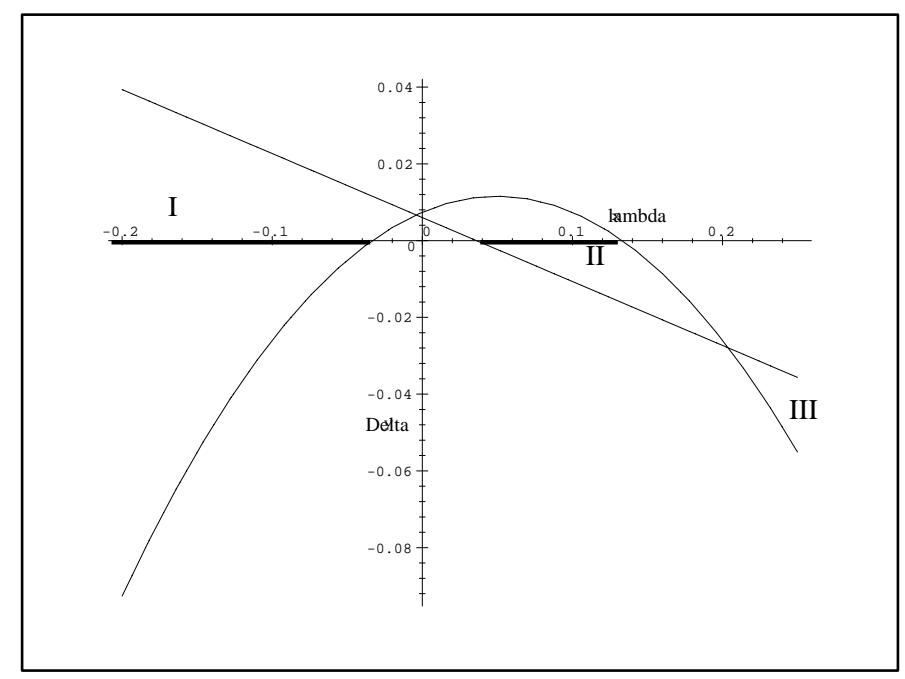

Figure 1: Existence of the resonance manifold in the presence of (scaled) detuning parameter $\Delta=\frac{\delta_{1}}{E_{0} a_{2}^{2}}$. The vertical axis represents $\Delta$ and the horizontal axis $\lambda=\frac{a_{1}}{3 a_{2}}$. The domain II and the unbounded domain I and III (both bounded by the parabola and the straight line) correspond with existence of the resonance manifold.

How is the effect of detuning in the case of existence of the resonance manifold? In the same way as before, in terms of parameters $\lambda$ and $\Delta=\delta_{1} /\left(E_{0} a_{2}^{2}\right)$, we can write for the existence of the resonant manifold

$$
0 \leq \frac{-360 \lambda+13-240 \Delta}{3600 \lambda^{2}-720 \lambda-3} \leq 1
$$

In figure 1, the area marked by I, II and III represent the domains of existence of the resonance manifold in the parameter space. The parabolic boundary of the domain represents the first normal mode $\left(q_{1}, p_{1}\right.$ direction $)$ and the straight line boundary the second normal mode. By fixing the detuning coefficient, we have a horizontal line on which we can move the resonance manifold from one normal mode to the other as we vary $\lambda$. The analysis can be repeated for fixed $\lambda$. The bold parts of the horizontal axes are the cases of exact resonance. Note that the intersection points are excluded as they correspond with the zero of the denominator in (15).

\subsection{The degeneration $\gamma_{2}=4 \gamma_{1}$}

Consider again the equations in (13). With the condition $\gamma_{2}=4 \gamma_{1}$, equations (13) become 


$$
\begin{aligned}
& \dot{\rho}_{1}=0+O\left(\varepsilon^{3}\right) \\
& \dot{\rho}_{2}=0+O\left(\varepsilon^{3}\right) \\
& \dot{\chi}=\varepsilon^{2}\left(2 \gamma_{1} E_{0}-2 \delta_{1}\right)+O\left(\varepsilon^{3}\right) .
\end{aligned}
$$

System (17) immediately yields that at exact resonance there will be no resonance manifold. Another consequence is that there exist a critical energy $E_{c}=\frac{\delta_{1}}{\gamma_{1}}$ such that the last equation of (17) is zero, up to order $\varepsilon^{3}$. It means we have to include even higher order terms of the hamiltonian in the analysis.

From the normal form (4), we know that this corresponds with the case that the normalized $H_{4}$ is zero. For the $1: 2$-resonance $H_{5}$ does not contain resonant terms. Thus the next nonzero term would be derived from $H_{6}$. As a consequence, the equations for amplitudes and phases are all of the same order, i.e. $O\left(\varepsilon^{4}\right)$. It is also clear from normal form (4) that in $H_{6}$ besides terms which represent interaction between two degrees of freedom (resonant terms), there are also interactions between each degree of freedom with itself (terms of the form $\tau_{1}{ }^{\alpha} \tau_{j}^{\beta}$ ).

To avoid a lengthy calculation and as an example, we consider a problem where $a_{1}=$ $a_{2}=0$. From the condition $\gamma_{2}=4 \gamma_{1}$ we derive $b_{2}=3 b_{1}+\frac{3}{16} b_{3}$. Then the last equation of (17) becomes

$$
\dot{\chi}=\varepsilon^{2}\left(\left(-\frac{3}{4} b_{1}+\frac{3}{64} b_{3}\right) \rho_{1}^{2}+4\left(-\frac{3}{4} b_{1}+\frac{3}{64} b_{3}\right) \rho_{2}^{2}-2 \delta_{1}\right)+O\left(\varepsilon^{3}\right) .
$$

Introducing the critical energy $E_{c}$, we have a degeneration of the last equation which gives an additional relation, i.e.

$$
\delta_{1}=\frac{1}{2}\left(\left(-\frac{3}{4} b_{1}+\frac{3}{64} b_{3}\right) \rho_{1}^{2}+4\left(-\frac{3}{4} b_{1}+\frac{3}{64} b_{3}\right) \rho_{2}^{2}\right) .
$$

We note also that for $\delta_{1}>0$ the critical energy exists providing $b_{1}<\frac{1}{16} b_{3}$.

We apply second order averaging to have an $O\left(\varepsilon^{2}\right)$ approximation on the time-scale $1 / \varepsilon^{4}$. We find for the approximations

$$
\begin{aligned}
\dot{\rho}_{1}= & -\varepsilon^{4} \frac{3}{32}\left(b_{1}{ }^{2}+\frac{5}{32} b_{1} b_{3}+\frac{3}{512} b_{3}{ }^{2}\right) \rho_{2}{ }^{2} \rho_{1}{ }^{3} \sin (\chi) \\
\dot{\rho}_{2}= & \varepsilon^{4} \frac{3}{128}\left(b_{1}{ }^{2}+\frac{5}{32} b_{1} b_{3}+\frac{3}{512} b_{3}{ }^{2}\right) \rho_{2} \rho_{1}{ }^{4} \sin (\chi) \\
\dot{\chi}= & \varepsilon^{4}\left(\frac{3}{64}\left(b_{1}{ }^{2}+\frac{5}{32} b_{1} b_{3}+\frac{3}{512} b_{3}{ }^{2}\right)\left(\rho_{1}^{4}-\frac{1}{8} \rho_{1}{ }^{2} \rho_{2}{ }^{2}\right) \cos (\chi)\right. \\
& +\frac{3}{64}\left(-4 b_{1}{ }^{2}+\frac{1}{2} b_{1} b_{3}+\frac{1}{256} b_{3}{ }^{2}\right) \rho_{1}^{4}+\frac{3}{64}\left(-46 b_{1}{ }^{2}+\frac{1}{4} b_{1} b_{3}+\frac{1}{128} b_{3}{ }^{2}\right) \rho_{1}{ }^{2} \rho_{2}{ }^{2} \\
& \left.+\frac{3}{64}\left(-44 b_{1}{ }^{2}+\frac{1}{2} b_{1} b_{3}+\frac{9}{64} b_{3}{ }^{2}\right) \rho_{2}{ }^{4}\right) .
\end{aligned}
$$

It is clear that the analysis of periodic solutions obtained by setting $\chi=0$ or $\chi=\pi$ runs along the same lines as in lower order resonance cases. Consider $\chi=0$. The fixed 
point of the averaged equations is determined by the last equation of (18). Since we are looking for periodic solutions which are different from normal modes, we assume both $\rho_{1}$ and $\rho_{2}$ to be nonzero. Writing $\xi=\left(\frac{\rho_{2}}{\rho_{1}}\right)^{2}$ we obtain a periodic solution by solving the quadratic equation

$$
a \xi^{2}+b \xi+c=0,
$$

where $a=-\frac{33}{16} b_{1}{ }^{2}+\frac{3}{128} b_{1} b_{3}+\frac{27}{4096} b_{3}{ }^{2}, b=-\frac{81}{32} b_{1}{ }^{2}-\frac{3}{64} b_{1} b_{3}-\frac{15}{8192} b_{3}{ }^{2}$ and $c=-\frac{9}{64} b_{1}{ }^{2}+$ $\frac{63}{2048} b_{1} b_{3}+\frac{15}{32768} b_{3}^{2}$. Assuming that $b_{3} \neq 0$, we have

$$
\begin{aligned}
& a=-\frac{33}{16} \kappa^{2}+\frac{3}{128} \kappa+\frac{27}{4096} \\
& b=-\frac{81}{32} \kappa^{2}-\frac{3}{64} \kappa-\frac{15}{8192} \\
& c=-\frac{9}{64} \kappa^{2}+\frac{63}{2048} \kappa+\frac{15}{32768},
\end{aligned}
$$

where $\kappa=\frac{b_{1}}{b_{3}}$. It is easy to see that $b<0$. Note that both the magnitude and the sign of $b_{3}$ is not important. We can also consider $\frac{b_{3}}{b_{1}}$ instead if $b_{3}=0$. We calculate the discriminant $D=b^{2}-4 a c$ and $-a, b$, and $c$ being quadratic in $\kappa$ - plot the function $D(\kappa)$ in figure 2.

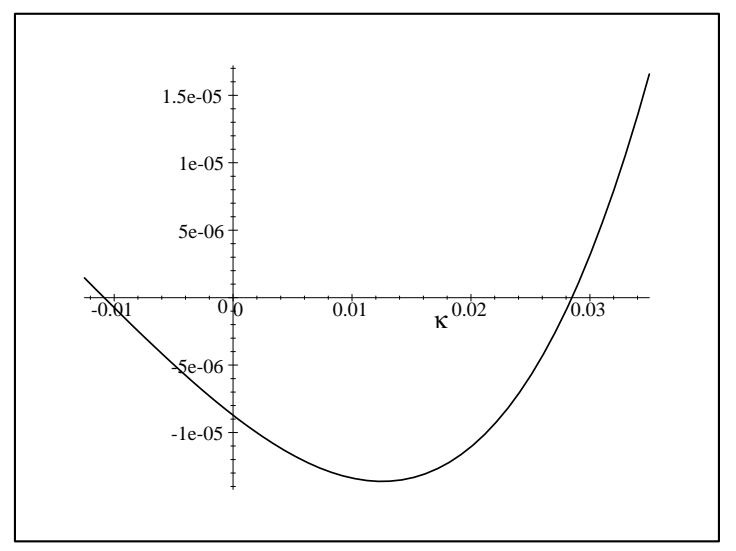

Figure 2: Plot of $D(\kappa)$. Positivity of $D(\kappa)$ is a necessary condition for periodic solutions to exist of system (9) with $\chi=0$, which are not normal modes.

There is an interval around $\kappa=0$ where the value of $D$ is negative. The value of $\kappa$ so that $D$ is zero can be calculated using numerics. Thus we know that except for small values of $\kappa$, we always have two roots for the quadratic equation (19). Knowing that we are looking for the root of equation (19) which is positive, we have to add another requirement. If we require $c / a$ to be positive and $b / a$ to be negative we will have two different periodic solutions. These requirements are satisfied by $\kappa \in\left(\frac{7}{64}-\frac{\sqrt{561}}{192}, \frac{1}{16}\right)$. When $\kappa$ is at the lower 
bound of the interval, the periodic solution coincides with the normal mode, in this case with the second normal mode. Note also that this interval contains the interval where the discriminant becomes zero or negative. The upper bound of the interval has to be excluded as $a$ vanishes there. Thus if $\kappa$ increases towards zero, the periodic solutions become closer, then coincide with each other and afterwards disappear. If we let $\kappa$ increase from zero, at some point a periodic solution will emerge and split up by increasing $\kappa$. For $\kappa \in\left(-\frac{9}{176}, \frac{7}{64}-\frac{\sqrt{561}}{192}\right)$ or $\left(\frac{1}{16}, \frac{7}{64}+\frac{\sqrt{561}}{192}\right)$ there is only one periodic solution. This is reasonable since one of the periodic solution coincides with one of the normal modes at the upper end points of each interval. It is easy to see that the case where $a$ vanishes corresponds to the existence of one periodic solution. For other values of $\kappa$ the periodic solution does not exist. Note that we are only considering the case $\chi=0$.

We have to apply the same reasoning to the other case and we expect conditions where there is no periodic solution (apart from the normal modes), one, two, three or four periodic solutions. Note that the analysis above also has to satisfy the existence condition for the critical energy, i.e. if $\delta>0$ the critical energy only exist for $\kappa<\frac{1}{16}$ and if $\delta<0$ for $\kappa>\frac{1}{16}$.

\section{The elastic pendulum}

In this section we will study one of the classical mechanical examples with discrete symmetry. Consider a spring with spring constant $s$ and length $l_{0}$, a mass $\mathrm{m}$ is attached to the spring; $g$ is the gravitational constant and $l$ is the length of the spring under load in the vertical position. The spring can both oscillate in the vertical direction and swing like a pendulum. This is called the elastic pendulum.

Let $r(t)$ be the length of the spring at time $t$ and $\varphi$ the angular deflection of the spring from its vertical position. In [10] van der Burgh uses a Lagrangian formulation to analyze the elastic pendulum, while in this paper we will use a hamiltonian formulation. The hamiltonian is given by

$$
H=\frac{1}{2 m}\left(p_{r}^{2}+\frac{p_{\varphi}^{2}}{r^{2}}\right)+\frac{s}{2}\left(r-l_{\circ}\right)^{2}-\operatorname{mgr} \cos \varphi,
$$

where $p_{r}=m \dot{r}$ and $p_{\varphi}=m r^{2} \dot{\varphi}$.

Introducing the elongation of the spring by $z=\frac{r-l}{l}$, we translate the origin of the coordinate system to the fixed point of the system where the elastic pendulum is hanging vertically at rest. By dividing by $l$ we normalize the length of the spring; we adjust also the momenta $p_{z}=l p_{r}$ to keep the hamiltonian structure. The hamiltonian in the new variables is

$$
H=\frac{1}{2 m l^{2}}\left(p_{z}^{2}+\frac{p_{\varphi}^{2}}{(z+1)^{2}}\right)+\frac{s l^{2}}{2}\left(z+\frac{l-l_{0}}{l}\right)^{2}-m g l(z+1) \cos \varphi
$$

Put $\alpha_{1}=\omega_{z} \sigma$ and $\alpha_{2}=\omega_{\varphi} \sigma$ where $\sigma=m l^{2}$. We transform $\bar{z}=\sqrt{\alpha_{1}} z$ and $\bar{\varphi}=\sqrt{\alpha_{1}} \varphi$. To preserve the hamiltonian structure we also transform $p_{z}=\sqrt{\alpha_{1}} \overline{p_{z}}$ and $p_{\varphi}=\sqrt{\alpha_{2}} \overline{p_{\varphi}}$. 
Expanding this hamiltonian the two leading terms of the hamiltonian are,

$$
\begin{aligned}
& H_{0}=\frac{1}{2} s\left(l-l_{\circ}\right)^{2}-m g l \\
& H_{1}=\frac{1}{\sqrt{\omega_{z} \sigma}}\left(s l\left(l-l_{\circ}\right)-m g l\right) z .
\end{aligned}
$$

We define the coordinate such that the pendulum is at rest in $\left(p_{z}, z, p_{\varphi}, \varphi\right)=(0,0,0,0)$. As a consequence the linear term of the hamiltonian is zero. Thus we have $s\left(l-l_{0}\right)=m g$. This condition restricts the ratio of the frequencies of the two oscillators, i.e. $\frac{\omega_{z}}{\omega_{\varphi}}>1$. The restriction is natural since at the equilibrium position the resultant force of gravitational force $(m g)$ and spring force $\left(s\left(l-l_{\circ}\right)\right)$ is zero. With $\sqrt{\frac{s}{m}}=\omega_{z}$ and $\sqrt{\frac{g}{l}}=\omega_{\varphi}$, the remaining terms in the expansion of the hamiltonian are

$$
\begin{aligned}
& H_{2}=\frac{1}{2} \omega_{z}\left(z^{2}+p_{z}^{2}\right)+\frac{1}{2} \omega_{\varphi}\left(\varphi^{2}+p_{\varphi}^{2}\right) \\
& H_{3}=\frac{\omega_{\varphi}}{\sqrt{\sigma \omega_{z}}}\left(\frac{1}{2} z \varphi^{2}-z p_{\varphi}^{2}\right) \\
& H_{4}=\frac{1}{\sigma}\left(\frac{3}{2} \frac{\omega_{\varphi}}{\omega_{z}} z^{2} p_{\varphi}^{2}-\frac{1}{24} \varphi^{4}\right) \\
& H_{5}=-\frac{1}{\sigma \sqrt{\sigma \omega_{z}}}\left(\frac{1}{24} z \varphi^{4}+2 \frac{\omega_{\varphi}}{\omega_{z}} z^{3} p_{\varphi}^{2}\right) \\
& H_{6}=\frac{1}{\sigma^{2} \omega_{\varphi}}\left(\frac{1}{720} \varphi^{6}+\frac{5}{2}\left(\frac{\omega_{\varphi}}{\omega_{z}}\right)^{2} z^{4} p_{\varphi}^{2}\right) \\
& H_{7}=\frac{1}{\sigma^{2} \omega_{\varphi} \sqrt{\sigma \omega_{z}}}\left(\frac{1}{720} z \varphi^{6}-3\left(\frac{\omega_{\varphi}}{\omega_{z}}\right)^{2} z^{5} p_{\varphi}^{2}\right)
\end{aligned}
$$

As expected, the - relatively few - terms in the hamiltonian are symmetric in the second degree of freedom and also in $p_{z}$. Due to the restriction of the frequency ratio above, we will not have the $1: \lambda$-resonances with $\lambda>1$. On the other hand, the symmetry condition on the second degree of freedom eliminates the $3: 1$-resonance as a lower order resonance. The next resonant term of this resonance arises from $H_{8}$. Thus, for lower order resonances, the remaining cases are the $2: 1$ - and, if we allow small detuning, the $1: 1$-resonance. The 2 : 1-resonance has been intensively studied, see [10] or [5] for references. This resonance is the one with resonant terms of the lowest degree.

As noted in [10], for the 1 : 1-resonance, second order averaging still gives only zero for both the amplitudes and the phases (this is not rendered correctly in [13]). It follows that the $1: 1$-resonance is also eliminated as a lower order resonance.

Introduce the transformation $z=r_{1} \cos \left(\omega_{z} t+\phi_{1}\right), p_{z}=-r_{1} \sin \left(\omega_{z} t+\phi_{1}\right), \varphi=r_{2} \cos \left(\omega_{\varphi} t+\right.$ $\left.\phi_{2}\right)$, and $p_{\varphi}=-r_{2} \sin \left(\omega_{\varphi} t+\phi_{2}\right)$. Assuming $\omega_{z} \neq 2 \omega_{\varphi}$ and rescaling with $\varepsilon$ as usual we find 
the second-order averaged equations for amplitudes and phases

$$
\begin{aligned}
\dot{\rho}_{1}= & 0+O\left(\epsilon^{3}\right) \\
\dot{\rho}_{2}= & 0+O\left(\epsilon^{3}\right) \\
\omega_{\varphi} \dot{\psi}_{1}-\omega_{z} \dot{\psi}_{2}= & -\epsilon^{2} \frac{3}{4} \frac{\left(\omega_{\varphi}-\omega_{z}\right)\left(\omega_{z}^{2}+\omega_{z} \omega_{\varphi}-3 \omega_{\varphi}^{2}\right)}{\left(\omega_{z}+2 \omega_{\varphi}\right)\left(2 \omega_{\varphi}-\omega_{z}\right) \sigma} \rho_{1}{ }^{2}+ \\
& \frac{1}{16} \frac{\left(\omega_{\varphi}-\omega_{z}\right)\left(\omega_{z}^{3}+13 \omega_{z}^{2} \omega_{\varphi}+20 \omega_{z} \omega_{\varphi}{ }^{2}-28 \omega_{\varphi}^{3}\right)}{\omega_{z}\left(\omega_{z}+2 \omega_{\varphi}\right)\left(2 \omega_{z}-\omega_{\varphi}\right)} \rho_{2}{ }^{2}+O\left(\epsilon^{3}\right),
\end{aligned}
$$

where $\rho_{1}$ and $\rho_{2}$ are the approximations of $r_{1}$ and $r_{2}, \psi_{1}$ and $\psi_{2}$ are the approximations of $\phi_{1}$ and $\phi_{2}$, respectively.

The resonance manifold is determined by the requirement that the right hand side of equation (23) vanishes. Except for the 1:1-resonance (we exclude the $2: 1$-resonance), there is no degeneration in the last equation of (23). This implies the resonance manifold exists for all resonances with $\omega_{z} / \omega_{\varphi}>(\sqrt{13}-1) / 2 \approx 1.30277 \ldots$ and perhaps for the $1: 1$-resonance.

We will now consider the most prominent higher order resonances which are possible for the elastic pendulum problem. We start with the $3: 2$ - and the $4: 1$-resonance. For both resonances we know that in general the resonant terms arises from $H_{5}$ which implies that the amplitude variation will be zero up till second order averaging. This is in agreement with (23). To determine which resonance in the elastic pendulum arises from $H_{5}$, we have to normalize.

\begin{tabular}{|c|c|c|c|}
\hline$\omega_{z}: \omega_{\varphi}$ & Resonant Part & $d_{\varepsilon}$ & Interaction time-scale \\
\hline $4: 1$ & $H_{5}$ & $\varepsilon^{1 / 2}$ & $\varepsilon^{-5 / 2}$ \\
$4: 3$ & $H_{7}$ & $\varepsilon^{3 / 2}$ & $\varepsilon^{-7 / 2}$ \\
$6: 1$ & $H_{7}$ & $\varepsilon^{3 / 2}$ & $\varepsilon^{-7 / 2}$ \\
$3: 1$ & $H_{8}$ & $\varepsilon^{2}$ & $\varepsilon^{-4}$ \\
$8: 1$ & $H_{9}$ & $\varepsilon^{5 / 2}$ & $\varepsilon^{-9 / 2}$ \\
$3: 2$ & $H_{10}$ & $\varepsilon^{3}$ & $\varepsilon^{-5}$ \\
\hline
\end{tabular}

Table 2: The table presents the most prominent higher order resonances of the elastic pendulum with lowest order resonant terms $H_{k}$. The third column gives the size of the resonance domain in which the resonance manifold is imbedded while in the fourth column we find the time-scale of interaction in the resonance domain.

For the $3: 2$-resonance we find

$$
\begin{aligned}
& \dot{\rho_{1}}=0+O\left(\epsilon^{4}\right) \\
& \dot{\rho_{2}}=0+O\left(\epsilon^{4}\right) \\
& \dot{\chi_{1}}=-\epsilon^{2} \frac{1}{336} \frac{108 \rho_{1}{ }^{2}-277 \rho_{2}{ }^{2}}{\sigma}+O\left(\epsilon^{4}\right),
\end{aligned}
$$

where $\chi_{1}=2 \psi_{1}-3 \psi_{2}$. 


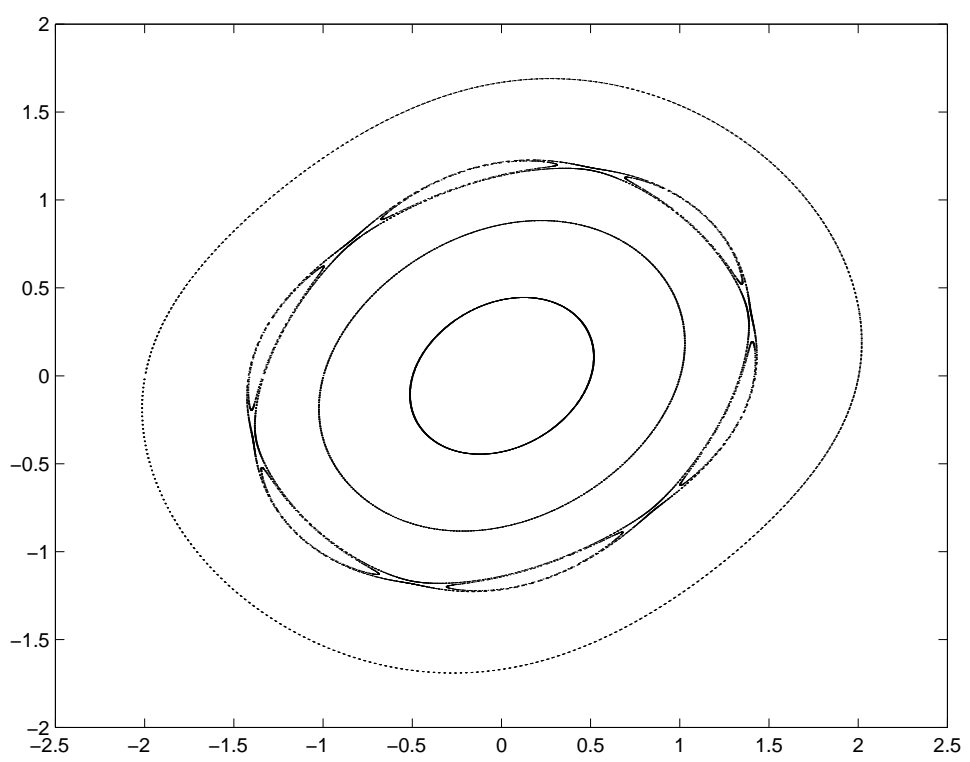

Figure 3: The Poincare map for the $6: 1$-resonance in the second degree of freedom ( $\varepsilon=0.75$ and the energy $E=5$; large values for illustration purposes). The saddles are connected by heteroclinic cycles and inside the cycles (islands) are centers.

For the $4: 1$-resonance the normalization produces

$$
\begin{aligned}
& \dot{\rho_{1}}=-\epsilon^{3} F_{1}\left(\rho_{1}, \rho_{2}\right) \sin \left(\chi_{2}\right)+O\left(\epsilon^{4}\right) \\
& \dot{\rho_{2}}=-\epsilon^{3} F_{2}\left(\rho_{1}, \rho_{2}\right) \sin \left(\chi_{2}\right)+O\left(\epsilon^{4}\right) \\
& \dot{\chi}_{2}=-\epsilon^{2} \frac{3}{64} \frac{68 \rho_{1}^{2}-27 \rho_{2}^{2}}{\sigma}-\epsilon^{3} F_{3}\left(\rho_{1}, \rho_{2}\right) \cos \left(\chi_{2}\right)
\end{aligned}
$$

where $\chi_{2}=\psi_{1}-4 \psi_{2} ; F_{1}, F_{2}$ and $F_{3}$ depend non-trivially on $\rho_{1}$ and $\rho_{2}$.

The result in (24) shows that for the $3: 2$-resonance, there is no resonant term in the normalized hamiltonian up to degree 5. In (25), which is for the $4: 1$-resonance, there are resonant terms in the normalized hamiltonian of degree 5. The conclusion is that, after the first-order $2: 1$-resonance, the $4: 1$-resonance is the most prominent resonance in the elastic pendulum. Following the analysis in section 3, we can also determine the sizes of the resonance manifolds which depend on the lowest degree of resonant terms in the normal form. We repeat this for cases in which the resonant terms arise in $H_{7}, \ldots, H_{10}$. The results are summarized in table 2 . Note that a low order resonance as the $3: 1$-resonance figures here at relatively high order.

We checked our result numerically for some of the resonances by constructing the Poincaré map and by calculating the size of the resonance domain. In the numerical integrations we vary $\varepsilon$ and study how this affects the size of the resonance manifold. We found confirmation for the $4: 1$-resonance and the $6: 1$-resonance. As table 2 shows, the 


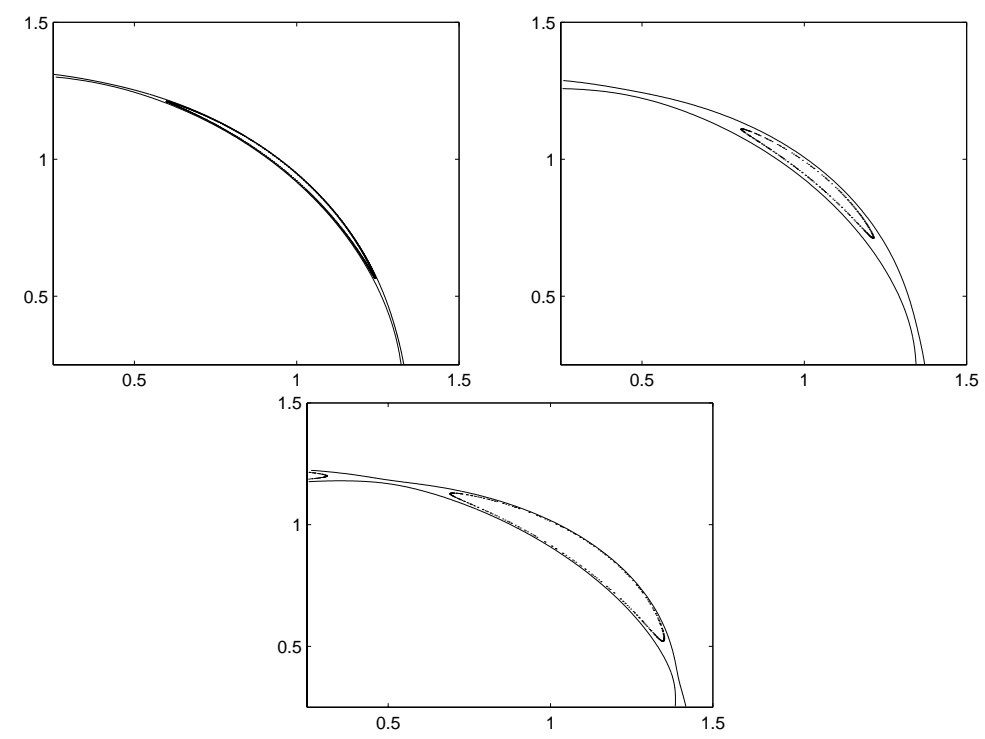

Figure 4: The 6:1-resonance. Part of the map in the second degree of freedom direction for several values of $\varepsilon$, the energy $E=5$. The top-left figure is for $\varepsilon=0.25$, the top-right figure is for $\varepsilon=0.5$ and the figure below is for $\varepsilon=0.75$.

numerical integration takes a long time. Figure 3 shows the map for the $6: 1$-resonance. To avoid long computation times, we increased the value of $\varepsilon$. In figure 4 we demonstrate the size and visibility of the resonance domain as $\varepsilon$ increases for the $6: 1$-resonance. In figure 5 the $4: 1$-resonance and the $6: 1$-resonance are compared.

\section{Conclusion and comments}

We have shown that symmetry assumptions strongly affect some of the lower order and higher order resonances in two degrees of freedom hamiltonian systems. In those cases, the symmetry assumption on one of the degrees of freedom implies a degeneration of the normal form. This degeneration forces us to extend the normalization. Consequently, the resonant terms appear at higher order compared with the case without symmetry assumptions. The conclusion is then that some of the lower order resonances behave like higher order ones. This makes sense since we know that for instance the $1: 2$ resonance can be viewed as $2: 4$ resonance or $3: 6$ resonance etc.

In the general, mathematically generic case, lower order resonance corresponds with strong interaction between the modes while higher order resonance corresponds with weak interaction. For symmetric potential problems in $1: 2$ resonance, we have shown that at a certain critical value of the energy, localized in phase-space at some distance of equilibrium, the system behaves like a strong resonance while for other values of the energy it produces higher order resonance. We note that the presence of this critical energy involves the 

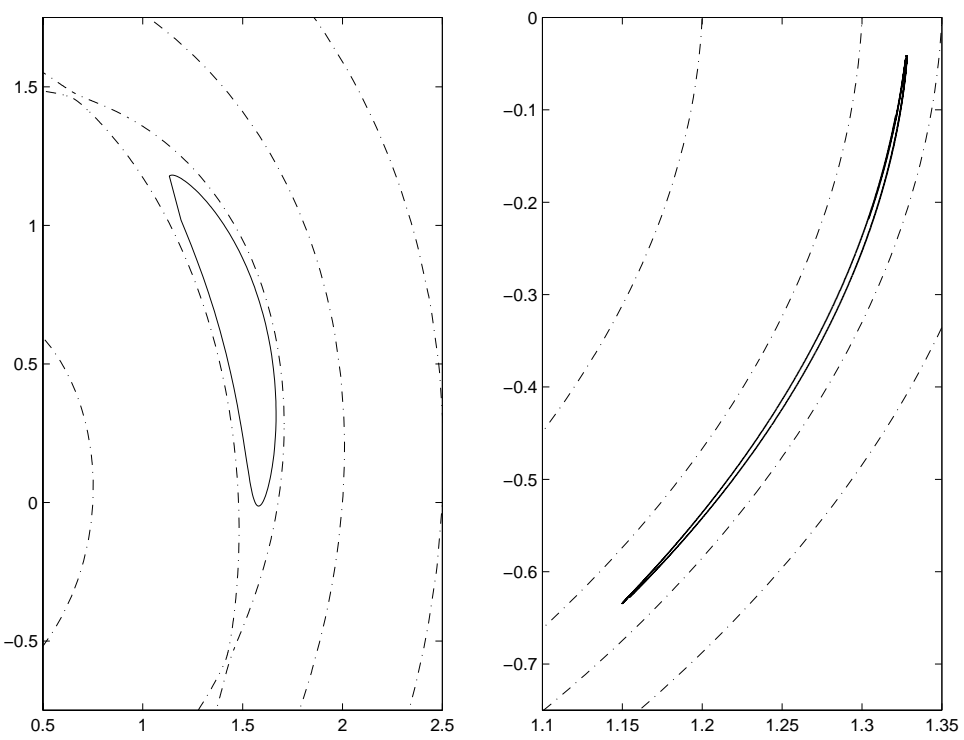

Figure 5: Part of the map in the second degree of freedom direction for the $4: 1$-resonance (left) and the $6: 1$-resonance (right); $\varepsilon=0.1$ and the energy $E=5$.

detuning parameter. More analysis is needed to see what part this critical energy may play in other systems.

In applying the analysis to the elastic pendulum we have found a numerical confirmation of our analytic estimates of the size of the resonance domain. Also we have found a new hierarchy in the resonances due to two reasons. First because of physical restrictions the $m: n$ resonances with $m<n$ are eliminated. Secondly the symmetry assumption. As is well-known the $2: 1$ resonance is the most prominent resonance, the next one is the $4: 1$ resonance. There are still some unsolved problems for the $1: 1$ resonance but we note that from the point of view of mechanics this resonance is of lesser importance for the elastic pendulum.

\section{Acknowledgments}

J. M. Tuwankotta thanks the Mathematisch Instituut, Universiteit Utrecht, the Netherlands and CICAT TUDelft, for financial support during the execution of the research; thanks to Lennaert van Veen, Menno Verbeek, Luis M. Artiles, Bob Rink and Martijn van Manen for many comments and discussion. J.M. Tuwankotta thanks Santi Goenarso for every support during a difficult time.

\section{References}

[1] Arnol'd, V.I., Mathematical Methods of Classical Mechanics, Springer-Verlag, New York etc., 1978. 
[2] Birkhoff, G.D., Dynamical Systems, reprinted by American Mathematical Society, 1972.

[3] Cicogna, G. Gaeta,G., Symmetry and Perturbation Theory in Nonlinear Dynamics, Lecture Notes in Physics, Springer-Verlag, 1999.

[4] Kozlov, V.V., Symmetries, Topology, and Resonances in Hamiltonian Mechanics, Ergebnisse der Mathematik und ihre Grenzgebiete 31, Springer-Verlag, 1996.

[5] Nayfeh, A.H., Mook, D.T., Nonlinear Oscillations, Wiley-Interscience, New York, 1979.

[6] Sanders, J.A., Are higher order resonances really interesting?, Celestial Mech. 16, pp. 421-440, 1978.

[7] Sanders, J.A., Verhulst, F., Approximations of Higher Order Resonances with an Application to Contopoulos' Model Problem, in Asymptotic Analysis, from theory to application,(F.Verhulst, ed.), Lecture Notes Math. 711, pp. 209-228, Springer-Verlag, Heidelberg etc. (1979).

[8] Sanders, J.A., Verhulst, F., Averaging Method on Nonlinear Dynamical System, Applied Math. Sciences 59, Springer-Verlag, New York etc., 1985.

[9] van den Broek, B., Studies in Nonlinear Resonance, Applications of Averaging, Ph.D. Thesis University of Utrecht, 1988.

[10] van der Burgh, A.H.P., On The Higher Order Asymtotic Approximations for the Solutions of the Equations of Motion of an Elastic Pendulum, Journal of Sound and Vibration 42, pp. 463-475, 1975.

[11] Verhulst, F., Discrete Symmetric Dynamical Systems at the Main Resonances with Applications to Axi-symmetric Galaxies, Phil. Trans. Royal Society London 290 pp. 435-465, 1979.

[12] Verhulst, F., Nonlinear Differential Equations and Dynamical Systems, 2nd ed., Springer Verlag, Berlin,1996.

[13] Verhulst, F., Symmetry and Integrability in Hamiltonian Normal Forms, in Symmetry and Perturbation Theory, D. Bambusi and G. Gaeta (eds), Quaderni GNFM pp. 245284, Firenze, 1998. 